\title{
VARIA LEXICOGRAPHICA GRAECA MANUSCRIPTA II: SACRA ET PROFANA*
}

The author discusses the identification made by G. de Andrés ( $C F C$ VI, I974, pp. 239-244) of two lexica in the Matritensis BU 30. On the basis of the comparison with several editions and catalogues a Lexicon Demosthenis and another epistolarum s. Pauli are identified.

E1 panorama de la Lexicografía griega tardía que recientemente ha trazado C. Serrano Aybar ${ }^{1}$ deja fuera, como es natural por su necesario esquematismo, muchos léxicos pequeños y sin demasiada importancia. El propósito de esta breve nota es aclarar, en torno a varios de estos léxicos, algunas cuestiones que se presentan en las descripciones de los manuscritos que los contienen y, en concreto, varias que se refieren a un testimonio de la Lexicografía griega tardía muy poco utilizado, el Matritense $\mathrm{BU} 30$ (del siglo $\mathrm{x}-\mathrm{xI})^{2}$. El léxico contenido en ff. $28 \mathrm{r}-292^{v}<$ lexicon vocum quae in Demosthenis orationibus Philippicis et deliberativis occurrunt $>\mathbf{3}$, pese a que G. de Andrés remite a las

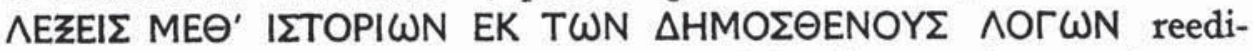
tadas recientemente por $\mathrm{K}$. Latte-H. Erbse ${ }^{5}$, no tiene nada que

* La primera de esta serie de notas, "Varia Lexicographica graeca manuscripta I: Voces animalium», en prensa en Habis VIII, 1977.

1 "Lexicografía antigua y medieval" en F. Rodriguez Adrados (et alii), Introducción a la Lexicografía griega, Madrid, 1977. Véase para más detalles L. Cohn, "Griechische Lexicographie", en K. Brugmann, Griechische Grammatik, Munich, I900, pp. 575-6I6 y Tolmkiehn, $R E$ XII 2, 1925, art. Lexikographie, cols. 2432$24^{82}$.

- Véase G. de Andrés, "Catálogo de los códices griegos de las colecciones: Complutense, Lázaro Galdiano y March, de Madrid», $C F C$ 6, r974, pp. 239-244.

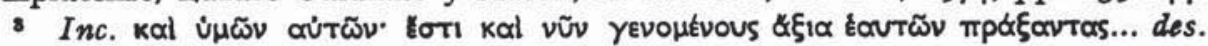

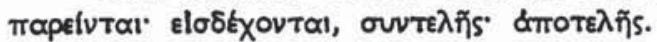

- $O$. c., p. $24 \mathrm{I}$.

- Lexica graeca minora, Hildesheim, 1965, pp. r40-165; se trata de un trabajo de I. Sakkelion publicado en $B C H$ r, 1877, pp. ro-16 y 137-154 recogiendo el texto contenido en Patmiacus 263. 
ver con ellas. Su contenido, versando indudablemente sobre la obra demosténica, es otro totalmente distinto $\mathrm{y}$, más bien, coincide, aunque sólo en parte, con otros dos pequeños léxicos titulados $\Phi_{1} \lambda_{1} \pi \pi ı$ I $^{2} \nu$

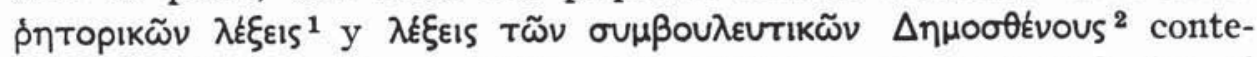
nidos en el Laurentianus LVII, 42 ff. $12 \mathrm{I}^{\mathrm{v}}$ y ss. y 133 y ss. ${ }^{3}$. Con el primero de estos dos léxicos A. B. Drachmann ${ }^{4}$ enumera 6 mss. en los

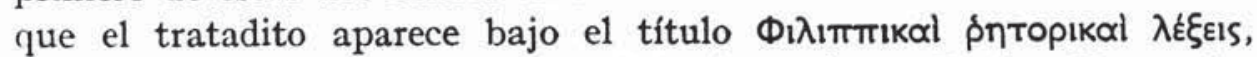
con igual comienzo, en orden no alfabético e sin II Bücher (Demosth., (Or. I-XI) eingeteilt» ${ }^{5}$. Por último, a esta misma serie podemos añadir

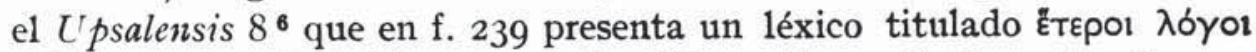

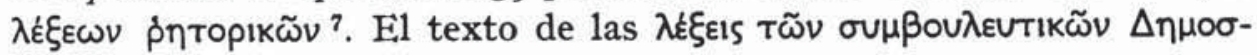
$\theta$ Évous es contenido igualmente en estos mismos manuscritus que citan Drachmann y Aurivillus, a excepción del Selestadiensis I0 $^{8}$.

En lo que se refiere a la interpretatio dictionum rhetoricarum contenida en los ff. 225-302 ${ }^{\mathrm{v}}$ del Matritensis ${ }^{9}$, De Andrés ${ }^{10}$ trae a cola-

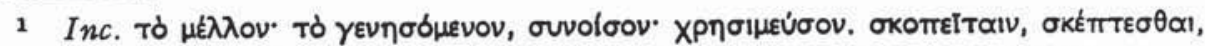

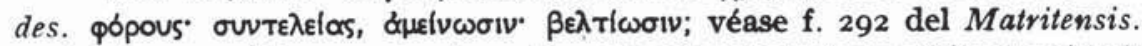

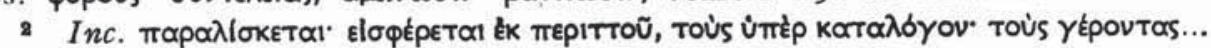

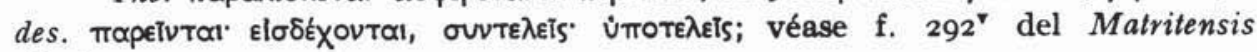
citado.

s Véase A. M. Randini, Catalogus codicum manuscriptorum Bibliothecae Mediceae Laurentianae, vol. II, Florencia, I768 (reimpresión, Leipzig, 196r), cols. $418-421$.

- "Die Ueberlieferung des Cyrillglossars", Det Kgl. Danske Videnskabernes Selskab. Historisk-filologiske Meddelelser XXI, 5, 1936, pp. 54 y 55.

s O. c., p. 56; son estos Vaticanis graecus 2130, Urbinas 157, Cryptensis $\mathrm{Z} \propto \mathrm{XXX}$, Holkhamiensis 289, Selestadiensis 105, Cephalleniensis monast. S. Gerasimi y añade uno, Vallicellianus $\mathrm{E} 37$, con el texto en orden alfabético. Recordemos que en Parisinus Suppl. graecus I 146 , ff. $40^{\nabla}-53^{v}$ aparece un ulexique des

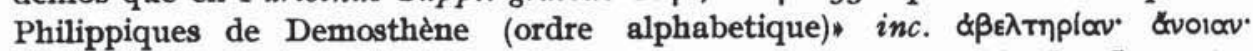

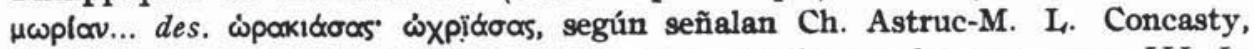
Bibliotheque nationale. Departement des mss. Catalogues des mss. grecs. III. Le supplément grec, tome III (901-137I), Paris, 1960, p. 294.

- Véase C. E. Aurivillus, Glossaria graeca manuscripta quae in Bibliotheca Reg. Academiae Upsaliensis asservantur, Upsala, 1822, p. 37. Citamos por la signatura que esta obra asigna a los mss. y no por las que éstos han adquirido posteriormente.

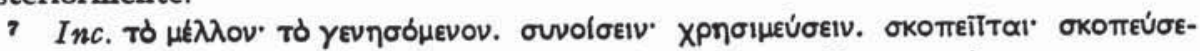

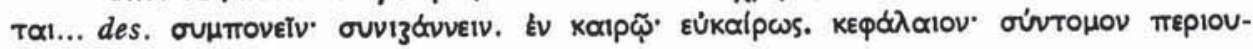

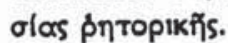

8 Se trata de un léxico no alfabético en tres libros y con 18 glosas en total.

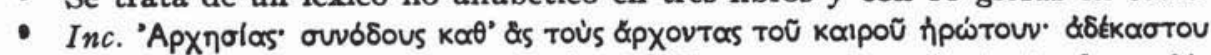

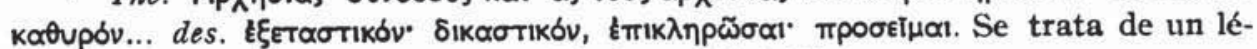
xico con términos de Demóstenes, Pindaro y Aristófanes.

10 O. c., p. $24 \mathrm{I}$. 
ción el Laurentianus LVII, 42 f. I29 que, esta vez sí, es el mismo texto. Esste léxico está contenido también en todos los mss. mencionados por Drachmann que hemos citado anteriormente $y$, aunque su título coincide un tanto con el quinto de los léxicos publicados por I.

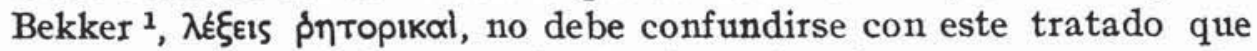
forma parte de los llamados Lexica Segueriana ${ }^{2}$; tengamos en cuenta, además, que en los mss. citados por Drachmann este léxico, la in-

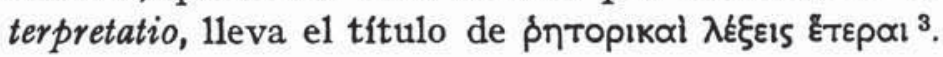

Finalmente, vamos a considerar ahora uno de los léxicos tocantes a libros sagrados que este mismo Matritensis BU 30 nos presenta en abundancia. La observación que debemos hacer toca al -lexicon epistolarum s. Pauli $>$ contenido en ff. 25I-257 que, como De Andrés nos dice 4, está también contenido en Vaticanus graecus 344 ff. $13 \mathrm{I}^{\mathrm{v}}-\mathrm{r} 34^{\mathrm{v}}$ y fue editado por J. Fr. Boissonade ${ }^{5}$. Por lo que respecta a esto último, una comparación del texto manuscrito y el impreso pone de relieve que son distintos, de forma que es éste otro pequeño detalle a tener en cuenta.

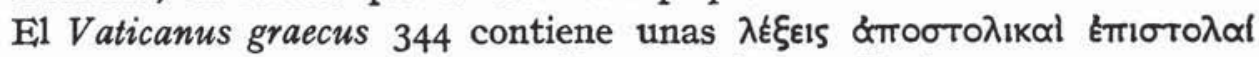
(sic) ${ }^{6}$ que parecen estar contenidas también en Vaticanus graecus 2130 f. I95 ${ }^{v} \mathrm{y}$, luego, en ff. $\mathrm{I} 34^{\mathrm{v}}-\mathrm{I} 35$ contiene una Epistola ad Hebraeos que

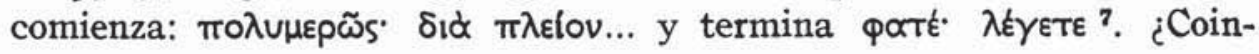

1 Anecdota graeca I, Berlín, 1814, pp. 195-318.

- Véase, en general, W. von Christ-W. Schmid-O. Stählin, Geschichte der griechischen Literatur. Zweiter Teil: die nachklassische Periode..., Munlch, 196r, p. 1093.

Otro léxico de parecido titulo y del que C. Serrano, o. c., p. 95, sólo men-

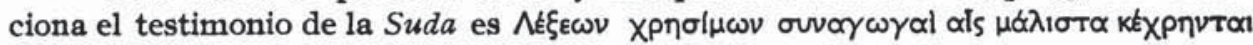

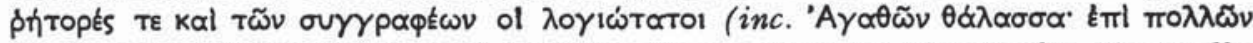

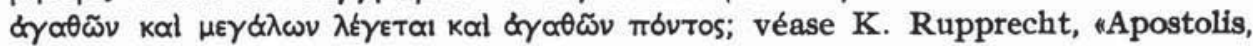
Eudem und Suidas", Philologus Suppl. XV, 1922 (Anhang III Excerpta ex Eudemi codice Parisino n. 2635 descripsit B. Niese), pp. I42-I6o e I. Bekker, o. c., III, pp.

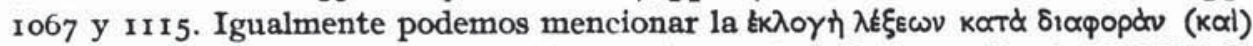

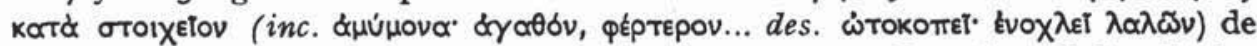
la que Drachmann, o. c., pp. 54-55, menciona 6 mss. Para la edición de las

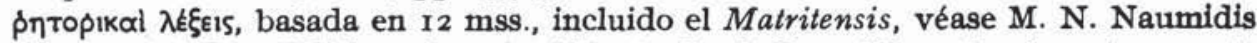

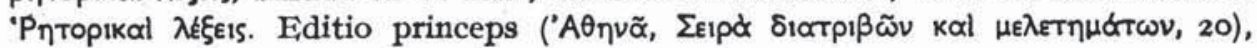
Atenas, 1975 .

- O. c., p. 240.

- Anecdota graeca e codicibus Regiis III, Paris, I831, pp. 339-353.

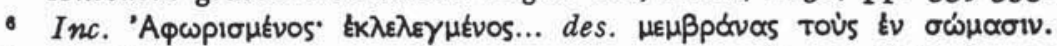

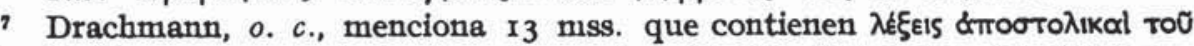
aylov Moúiou con un texto igual en inc. y des. al total de los dos del Vaticanus graecus 344 . Por su parte Aurivillus, o.c., p. 28 encuentra en el citado Upsalensis 8 , f. 198 un léxico sin título que contiene voces ex epistolis Paullinis hoc ordine, 
ciden estos textos con los del Matritensis realmente? Vamos a verlo.

Comienza éste (f. 25I) por $A d$ Romanos (título en latín de letra re-

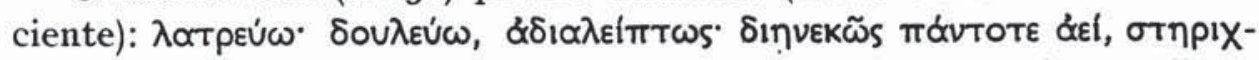

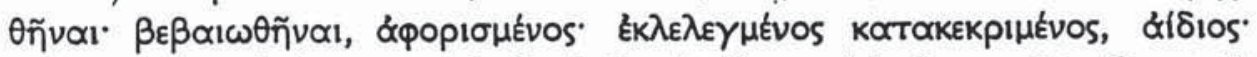

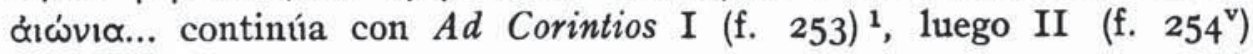
y siguen otras epístolas más brevemente tratadas hasta llegar, en f. 256, al final: $\mu \varepsilon \mu \beta p \alpha \alpha v \alpha s^{\circ}$ тоĩs $\varepsilon^{2} \nu \sigma \omega \mu \alpha \sigma$. Empieza en este punto Ad Hebraeos

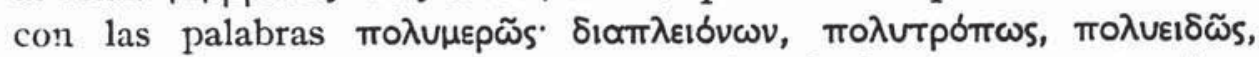

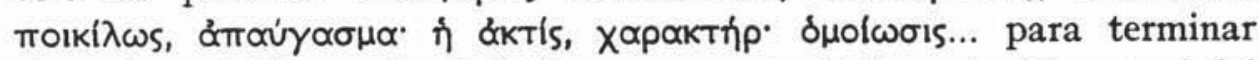
(f. 257) $\propto \tau \tau \dot{\varepsilon} \cdot \lambda \varepsilon \gamma \varepsilon \tau \varepsilon$. En definitiva, pues, este léxico coincide con el del Vaticanus graecus 344 , pero no tiene nada que ver con las EPMHNEIAI

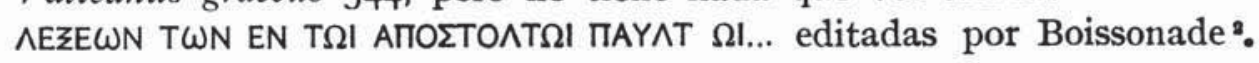
Por supuesto, no hemos pretendido dar aquí una lista exhaustiva de los testimonios manuscritos que tocan a las obras citadas ni tampoco aclarar otras cuestiones de fondo; por otro lado, la brevedad de las noticias contenidas en los diversos catálogos dificulta la precisión de detalles que, la verdad sea dicha, tan sólo garantizamos para los pasajes referidos al Matritensis BU 30 que hemos manejado. Hemos respetado, también debemos decirlo, la ortografía de los catálogos y testimonios manuscritos.

\section{Antonio Bravo García}

\footnotetext{
Corinth. Thess. Galat. Ephes. Collos. Rom. Timoth. Tit. et Phil. (inc. тоบ̃ $\theta \in \mathrm{E}$

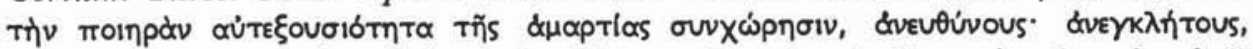
\&mpóry

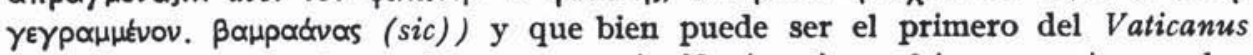

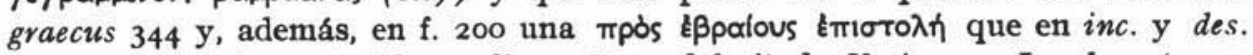
corresponde a la contenida en ff. $134^{\mathrm{N}}-\mathrm{r} 35$ del citado Vaticanus. Igualmente, en el Laurentianus LVII $4^{2}$ se contienen unas voces ex Epistolis D. Pauli como recoge Bandini y lo mismo en el Parisinus Suppl. graecus 1146, ff. 67-70, Leidensis Bibl. Publ. 44I, Vossianus misc. 20, Vossianus graecus $Q 63$, ff. $168^{\top}-172$ y otros muchos más.

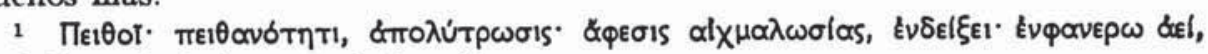

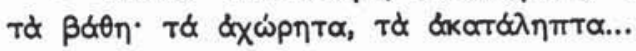

